\title{
THE RADON-NIKODYM THEOREM AND THE MEAN CONVERGENCE OF BANACH SPACE VALUED MARTINGALES
}

\author{
J. J. UHL, JR. ${ }^{1}$
}

In 1943, Phillips [3] extended the classical Radon-Nikodym Theorem to the setting of Banach space valued set functions. Later, in the early sixties, Chatterji [1] and Scalora [4] proved a theorem dealing with the norm convergence of martingales in the $L^{p}(1 \leqq p<\infty)$ of Banach space valued functions. Even though there has been much discussion dealing with the relationships between martingale convergence and derivations, there seems to be no explicit statement of the fact that the Chatterji-Scalora mean martingale convergence theorem can be deduced directly from Phillips' Theorem. Equally, there appears to be no mention that a strengthened version of the ChatterjiScalora theorem directly implies (an important special case of) Phillips' Radon-Nikodym Theorem. The purpose of this note is to demonstrate these facts.

Throughout this note, $(\Omega, \Sigma, \mu)$ is a fixed finite measure space; $\mathfrak{X}$ is a Banach space, and $L^{p}(\Omega, \Sigma, \mu, \mathfrak{X})\left(=L^{p}(\mathfrak{X})\right), 1 \leqq p<\infty$, is the Banach space of all measurable $\mathfrak{X}$-valued (equivalence classes of) functions $f$ defined on $\Omega$ which satisfy $\|f\|_{p}=\left(\int_{\Omega}\|f\| p d \mu\right)^{1 / p}<\infty$. By a partition $\pi=\left\{E_{n}\right\}_{n=1}^{m}$ of $\Omega$, we shall mean a finite set of disjoint sets in $\Sigma$ such that $\bigcup_{n=1}^{m} E_{n}=\Omega$.

Definition. An additive set function $F$ defined on $\Sigma$ and having its values in $\mathfrak{X}$ is said to be of $p$-bounded variation $(1 \leqq p<\infty)$ if

$$
\|F\|_{p}=\left(\sup _{\pi} \sum_{\pi}\left(\frac{\left\|F\left(E_{n}\right)\right\|}{\mu\left(E_{n}\right)}\right)^{p} \mu\left(E_{n}\right)\right)^{1 / p}
$$

where the supremum is taken over all partitions $\pi=\left\{E_{n}\right\}$ and the convention $0 / 0=0$ is observed here and throughout.

The following is a special, but important, case of Phillips' generalization of the Radon-Nikodym Theorem.

ThEOREM I [3]. If $\mathfrak{X}$ is reflexive and $F: \Sigma \rightarrow \mathfrak{X}$ is countably additive, $\mu$-continuous, and of $p$-bounded variation $(1 \leqq p<\infty)$, then there exists $f \in L^{p}(\mathfrak{X})$ such that $F(E)=\int_{E} f d \mu$ for all $E \in \Sigma$, where the integral is the Bochner integral.

Received by the editors April 1, 1968.

1 This research was supported in part by NSF Grant GP-55183 to Carnegie Institute of Technology. 
Leaving, for the time being, the Radon-Nikodym-Phillips Theorem, we shall now establish the setting for a generalization of the Chatterji-Scalora mean martingale convergence theorem.

Definition. Let $T$ be a directed set and $\left\{B_{t}, t \in T\right\}$ be an increasing net of sub- $\sigma$-fields of $\Sigma$ (i.e. if $t_{1}, t_{2} \in T$ and $t_{1} \leqq t_{2}$, then $B_{t_{1}} \subset B_{t_{2}}$ ). $\left\{f_{t}, B_{t}, t \in T\right\}$ is an $\mathfrak{X}$-valued martingale in $L^{p}(\mathfrak{X})$ if $f_{t} \in L^{p}(\mathfrak{X})$ for all $t \in T$, and if $t, t_{0} \in T$ and $t \geqq t_{0}$, then $E^{B_{t_{0}}}\left(f_{t}\right)=f_{t_{0}}$, where $E^{B_{t_{0}}}$ is the conditional expectation operator relative to $B_{t_{0}}$.

Here we understand that if $B$ is a sub- $\sigma$-field of $\Sigma$ and $f \in L^{p}(\mathfrak{X})$, $E^{B}(f)$ is the unique member of $L^{p}(\mathfrak{X})$ which is $B$-measurable and satisfies

$$
\int_{E} E^{B}(f) d \mu=\int_{E} f d \mu \quad \text { for all } E \in B
$$

The following theorem, in the case that the directed set $T$ is the positive integers with the natural order, is due to Chatterji [1] and Scalora [4]. It is the aim of this note to demonstrate the equivalence of the following theorem and Theorem I.

Theorem II. Let $\mathfrak{X}$ be reflexive and let $\left\{f_{t}, B_{t}, t \in T\right\}$ be an $\mathfrak{X}$-valued martingale in $L^{p}(\mathfrak{X})$.

(a) If $1<p<\infty$ and there exists $M$ such that $\left\|f_{t}\right\|_{p} \leqq M$ for all $t \in T$, then the net $\left\{f_{t}, t \in T\right\}$ converges in the $L^{p}(\mathfrak{X})$ norm.

(b) In the case $p=1$, if there exists a constant $M$ such that $\left\|f_{t}\right\|_{1} \leqq M$ for all $t \in T$ and a parameter $t_{0} \in T$ such that for any $\epsilon>0$ there corresponds $a \delta>0$ such that $t \geqq t_{0}$ and $\mu(E)<\delta$ implies $\int_{E}\left\|f_{t}\right\| d \mu<\epsilon$, then the net $\left\{f_{t}, t \in T\right\}$ converges in the $L^{1}(\mathfrak{X})$ norm.

We shall show that Theorems I and II are equivalent.

$\mathrm{I} \rightarrow$ II. Assume first that $1<p<\infty$. By the Hölder inequality and the fact that $\left\|f_{t}\right\|_{p} \leqq M$ for $t \in T$, we have, for $p^{-1}+q^{-1}=1$, and $E \in \Sigma$,

$$
\int_{E}\left\|f_{t}\right\| d \mu \leqq\left\|f_{t}\right\|_{p}\left\|\chi_{E}\right\|_{q} \leqq M\left\|\chi_{E}\right\|_{q}
$$

( $\chi_{E}$ is the indicator function of $E$.) Since for $1 \leqq q<\infty, \lim _{\mu(E) \rightarrow 0}\left\|\chi_{E}\right\|_{q}$ $=0$, it follows that for any $\epsilon>0$, there exists a $\delta>0$ such that $\mu(E)<\delta$ implies $\int_{E}\left\|f_{t}\right\| d \mu$ for all $t \in T$.

Now let $1 \leqq p<\infty$. Since $\left\{B_{t}, t \in T\right\}$ is an increasing net of sub- $\sigma$ fields of $\Sigma$, it follows easily that $U_{t \in T} B_{t}$ is a subfield of $\Sigma$ and hence generates a sub- $\sigma$-field, $\Sigma_{0}$, of $\Sigma$. Now we shall define an $\mathfrak{X}$ valued set function $F$ on $\Sigma_{0}$ by 


$$
F(E)=\lim _{z} \int_{E} f_{t} d \mu, \quad E \in \Sigma_{0} .
$$

Showing that such a set function exists requires some discussion. To show that $\lim _{t} \int_{E} f_{t} d \mu$ exists strongly in $\mathfrak{X}$ for each $E \in \Sigma_{0}$, choose $t_{0} \in T$ such that given any $\epsilon>0$ there exists a $\delta(=\delta(\epsilon))>0$ such that $t \geqq t_{0}$ implies $\int_{E}\left\|f_{t}\right\| d \mu<\epsilon$ whenever $\mu(E)>\delta$. In the case $p=1$, the existence of such a $t_{0}$ is guaranteed by hypothesis. In the case $1<p<\infty$, as the beginning of the proof shows, any $t \in T$ will qualify as the $t_{0}$ above. Now, let $\epsilon>0$ be fixed, but arbitrary, and choose $\delta>0$ such that $\int_{E}\left\|f_{t}\right\| d \mu<\epsilon / 4$ for $t \geqq t_{0}$ and $\mu(E)<\delta$. If $E \in \Sigma_{0}$, according to [2, III. 7.1], there exists a set $S \in \cup_{t \in T} B_{t}$ such that both $\mu(E-S)$ and $\mu(S-E)<\delta$. Since $S \in \cup_{t \in T} B_{t}$ and $\left\{B_{t}, t \in T\right\}$ is an increasing net, there exists $t^{\prime} \geqq t_{0}$ such that $S \in B_{t}$ for all $t \geqq t^{\prime}$. Moreover, for $t_{1}, t_{2} \geqq t^{\prime}$,

$$
\int_{S} f_{t_{i}} d \mu=\int_{S} E^{B t^{\prime}}\left(f_{t_{i}}\right) d \mu=\int_{S} f_{t^{\prime}} d \mu, \quad i=1,2,
$$

since $\left\{f_{t}, B_{t}, t \in T\right\}$ is a martingale and $S \in B_{t^{\prime}}$. Thus for $t_{1}, t_{2} \geqq t^{\prime}$ the following inequalities hold:

$$
\begin{aligned}
\left\|\int_{E} f_{t_{1}} d \mu-\int_{E} f_{t_{2}} d \mu\right\| \leqq & \left\|\int_{E} f_{t_{1}} d \mu-\int_{S} f_{t_{1}} d \mu\right\| \\
& +\left\|\int_{S} f_{t_{1}} d \mu-\int_{S} f_{t_{3}} d \mu\right\| \\
& +\left\|\int_{S} f_{t_{2}} d \mu-\int_{E} f_{t_{2}} d \mu\right\| \\
\leqq & \int_{E-S}\left\|f_{t_{1}}\right\| d \mu+\int_{S-E}\left\|f_{t_{1}}\right\| d \mu \\
& +\int_{E-S}\left\|f_{t_{2}}\right\| d \mu+\int_{S-E}\left\|f_{t_{2}}\right\| d \mu,
\end{aligned}
$$

by the triangle inequality and $\left({ }^{*}\right)$ above,

$$
<4(\epsilon / 4)=\epsilon,
$$

by the choice of $t_{0}$ and $S$.

It follows that the set function $F$ as defined above is well defined on $\Sigma_{0}$. Moreover, according to [2, IV.10.6], $F$ is countably additive on $\Sigma_{0}$. In addition, $F$ clearly vanishes on $\Sigma_{0}$-sets of zero $\mu$-measure. Thus, by a result of Pettis, [2, IV.10.1 ], $F$ is $\mu$-continuous on $\Sigma_{0}$.

Next we shall show that $F$ is of $p$-bounded variation. 


$$
\begin{aligned}
& \|F\|_{p}^{p}=\sup _{\pi} \sum\left\|F\left(E_{n}\right)\right\|^{p} \mu\left(E_{n}\right)^{1-p} \\
& \left.=\sup _{\pi} \lim _{\imath} \sum_{\pi}\left\|\int_{E_{n}} f_{t} d \mu\right\| \|^{p}{ } E_{n}\right)^{1-p}, \text { by the definition of } F \text {, } \\
& \leqq \sup _{\pi} \lim _{t} \sup \sum_{\pi}\left\|f_{t} \chi_{E_{n}}\right\|_{p}^{p}\left\|\chi_{E_{n}}\right\|_{q}^{p} \mu\left(E_{n}\right)^{1-p} \text {, } \\
& \text { by the Hölder inequality with } p^{-1}+q^{-1}=1 \text {, } \\
& =\sup _{x} \lim \sup _{t} \sum_{\pi}\left\|f_{t} \chi_{E_{n}}\right\|_{p \mu}^{p}\left(E_{n}\right)^{p / q+1-p} \text {, where if } q=\infty, p / q=0 \text {, } \\
& =\sup _{\pi} \lim \sup _{t} \sum_{\pi} \int_{E_{n}}\left\|f_{t}\right\|^{p} d \mu \text {, since } p / q+1-p=0 \text {, } \\
& =\sup _{\pi} \lim \sup _{t} \int_{\Omega}\left\|f_{t}\right\|^{p} d \mu \leqq M^{p} \text {. }
\end{aligned}
$$

Therefore the hypothesis of Theorem I is satisfied by $F$. By Theorem I, there exists a $\Sigma_{0}$-measurable $f \in L^{p}(\mathfrak{X})$ such that $F(E)=\int_{E} f d \mu$ for all $E \in \Sigma_{0}$.

To finish the proof, we shall show that $\lim _{t} f_{t}=f$ in the $L^{p}(\mathfrak{X})$ norm. First note that if $t_{0} \in T$ is arbitrary and $t \geqq t_{0}$, by the fact that $\left\{f_{t}, B_{t}, t \in T\right\}$ is a martingale,

$$
\int_{E} f_{t} d \mu=\int_{E} E^{B_{t_{0}}}\left(f_{t}\right) d \mu=\int_{E} f_{t_{0}} d \mu
$$

for all $E \in B_{t_{0}}$. From the definition of $F$ and $f$, it follows immediately that $\int_{E} f d \mu=\int_{E} f_{t_{0}} d \mu$ for all $E \in B_{t_{0}}$. Accordingly, since $f_{t_{0}}$ is $B_{t_{0}}$ measurable, $E^{\boldsymbol{B}_{t_{0}}}(f)=f_{t_{0}}$. Moreover, if $\epsilon>0$ is given, the $\Sigma_{0 \text {-measur- }}$ ability guarantees, by [2, III.8.3], the existence of a simple function $g=\sum_{i=1}^{m} x_{i} \chi_{E_{i}}, x_{i} \in \mathfrak{X}, E_{i} \in U_{t \in T} B_{\imath}$ such that $\|f-g\|_{p}<\epsilon / 2$. Now since $\left\{B_{t}, t \in T\right\}$ is an increasing net of sub- $\sigma$-fields of $\Sigma$, there exists a $\bar{t} \in T$ such that $E_{i} \in B_{t}$ for all $t \geqq \bar{t}$ and all $i=1, \cdots, m$. Therefore $E^{B_{t}}(g)=g$ for all $t \geqq \bar{t}$. Hence for $t \geqq \bar{t}$, we have

$$
\begin{aligned}
& \left\|f-f_{t}\right\|_{p} \leqq\|f-g\|_{p}+\left\|g-f_{t}\right\|_{p} \\
& =\|f-g\|_{p}+\left\|E^{B_{t}}(g-f)\right\|_{p}, \\
& \text { since } E^{B_{t}}(f)=f_{t} \text { and } E^{B_{t}}(g)=g \text {, for all } t \geqq t_{0} \text {, } \\
& \leqq 2\|f-g\|_{p} \text {, since } E^{B_{t}} \text { is a contraction, } \\
& \leqq 2(\epsilon / 2)=\epsilon, \text { by the choice of } g \text {. }
\end{aligned}
$$

This proves $\mathrm{I} \rightarrow \mathrm{II}$. 
$\mathrm{II} \rightarrow \mathrm{I}$. Assume first that $1<p<\infty$ and let $F$ satisfy the hypothesis of Theorem I. For each partition $\pi=\left\{E_{n}\right\}$, form the simple function

$$
f_{\pi}=\sum_{\pi} \frac{F\left(E_{n}\right)}{\mu\left(E_{n}\right)} \chi_{E_{n}} .
$$

If $\sigma(\pi)$ denotes the (trivial) $\sigma$-field generated by $\pi=\left\{E_{n}\right\}$, and the collection $P$ of all partitions is directed by the partial ordering of refinement, then $\left\{f_{\pi}, \sigma(\pi), \pi \in P\right\}$ becomes a martingale. Moreover,

$$
\begin{aligned}
\left\|f_{\pi}\right\|_{p} & =\left(\int_{\Omega}\left\|\sum_{\pi} \frac{F\left(E_{n}\right)}{\mu\left(E_{n}\right)} \chi_{E_{n}}\right\| d \mu\right)^{1 / p} \\
& =\left(\sum_{\pi}\left\|\frac{F\left(E_{n}\right)}{\mu\left(E_{n}\right)}\right\|^{p} \mu\left(E_{n}\right)\right)^{1 / p} \leqq\|F\|_{p} .
\end{aligned}
$$

Thus the hypothesis of Theorem II is satisfied. By Theorem II, there exists $f \in L^{p}(\mathfrak{X})$ such that $\lim _{\pi}\left\|f_{\pi}-f\right\|_{p}=0$. Now we shall show that $f$ is the Radon-Nikodym-Phillips derivative of $F$. If $E \in \Sigma$,

$$
\begin{aligned}
\left\|F(E)-\int_{E} f d \mu\right\| & \leqq \\
& +\lim \sup _{\pi}\left\|F(E)-\int_{\boldsymbol{E}} f_{\pi} d \mu\right\| \\
& +\int_{E} f_{\pi} d \mu-\int_{E} f d \mu \| .
\end{aligned}
$$

The first term on the right is zero since if $E \in \pi_{0}$ and $\pi \geqq \pi_{0}, \int_{E} f_{\pi} d \mu$ $=F(E)$. On the other hand, by the Hölder inequality,

$$
\begin{aligned}
\lim \sup \left\|\int_{\boldsymbol{E}}\left(f_{\pi}-f\right) d \mu\right\| & \leqq \limsup _{\pi} \int_{E}\left\|f_{\pi}-f\right\| d \mu \\
& \leqq \lim \sup _{\pi}\left\|f_{\pi}-f\right\|_{p}\left\|\chi_{E}\right\|_{q}\left(p^{-1}+q^{-1}=1\right)=0 .
\end{aligned}
$$

by Theorem II. Thus $F(E)=\int_{E} f d \mu$ for all $E \in \Sigma$. This proves II $\rightarrow \mathrm{I}$ in the case $1<p<\infty$.

The $L^{1}(\mathfrak{X})$ case will be proved by use of the $L^{2}(\mathfrak{X})$ case. Let $F: \Sigma \rightarrow \mathfrak{X}$ be $\mu$-continuous, countably additive, and of bounded variation. By [3, Lemma 5.4], there exists a sequence $\left\{S_{n}\right\} \subset \Sigma$ such that $S_{n} \supset S_{n+1}$ and $\lim _{n \rightarrow \infty} \mu\left(S_{n}\right)=0$ and such that $\|F(E)\| \leqq n \mu(E)$ for all $E \in \Omega-S_{n}$. If $F_{n}$ is defined by $F_{n}(E)=F\left(E-S_{n}\right)$, then clearly $\left\|F_{n}\right\|_{2}<\infty$ for all $n$. Now if $f_{n}$ is the $L^{2}(\mathfrak{X})$-Radon-Nikodym-Phillips derivative of $F_{n}$, and we define $f$ on $\Omega$ by $f(s)=f_{n}(s)$ for $s \in S_{n-1}-S_{n}$ where $S_{0}=\Omega$, then $f$ is measurable. Moreover, since $F$ is $\mu$-continuous and $\lim _{n \rightarrow \infty} \mu\left(S_{n}\right)=0$, 
it follows that $F(E)=\lim _{n \rightarrow \infty} F\left(E-S_{n}\right)=\lim _{n \rightarrow \infty} F_{n}(E)=\lim _{n \rightarrow \infty} \int_{E} f_{n} d \mu$ $=\int_{E} f d \mu$. Also, since $F$ is of bounded variation, we have $\int_{\Omega}\|f\| d \mu$ $=\|F\|_{1}<\infty$, and $f \in L^{1}(\mathfrak{X})$. Q.E.D.

\section{REFERENCES}

1. S. D. Chatterji, $A$ note on the convergence of Banach space valued martingales, Math. Ann. 153 (1964), 142-149.

2. N. Dunford and J. T. Schwartz, Linear operators, Part I, Interscience, New York, 1958.

3. R. S. Phillips, On weakly compact subsets of a Banach space, Amer. J. Math. 65 (1943), 108-136.

4. F. S. Scalora, Abstract martingale convergence theorems, Pacific J. Math. 11 (1961), 347-374.

CARnegie-Mellon University 